\title{
Node-Positive Bladder Cancer After Neoadjuvant Chemotherapy Followed by Radical Cystectomy: A Single-Center Retrospective Study
}

\author{
Jang Ho Cho ${ }^{1,2}$, Ghee-Young Kwon ${ }^{3}$, Minyong Kang ${ }^{4}$, Hyun Hwan Sung ${ }^{4}$, \\ Hwang Gyun Jeon ${ }^{4}$, Byong Chang Jeong ${ }^{4}$, Seong II Seo ${ }^{4}$, Seong Soo Jeon ${ }^{4}$, \\ Hyun Moo Lee ${ }^{4}$, Han-Yong Choi ${ }^{4,5}$, Su Jin Lee ${ }^{1,6}$, Se Hoon Park ${ }^{1}$ \\ ${ }^{1}$ Division of Hematology-Oncology, Department of Medicine, Samsung Medical Center, Sungkyunkwan \\ University School of Medicine, Seoul, Korea \\ ${ }^{2}$ Division of Oncology, Department of Internal Medicine, Incheon St. Mary's Hospital, The Catholic University \\ of Korea, Incheon, Korea \\ ${ }^{3}$ Department of Pathology, Samsung Medical Center, Sungkyunkwan University School of Medicine, Seoul, Korea \\ ${ }^{4}$ Department of Urology, Samsung Medical Center, Sungkyunkwan University School of Medicine, Seoul, Korea \\ ${ }^{5}$ Department of Urology, Kangbuk Samsung Hospital, Seoul, Korea \\ ${ }^{6}$ Division of Hematology-Oncology, Department of Internal Medicine, Ewha Womans University College of \\ Medicine, Seoul, Korea
}

\begin{abstract}
Purpose: There remains a lot of unmet need to increase understanding of node-positive (ypN+) muscle invasive bladder cancer (MIBC) after neoadjuvant chemotherapy and radical cystectomy to decide the appropriate therapeutics. Materials and Methods: In a retrospective study using the center cancer chemotherapy registry, we found 113 MIBC patients who were treated with neoadjuvant chemotherapy involving gemcitabine and cisplatin (GP) followed by radical cystectomy between 2010 and 2014. Disease-free survival (DFS) and overall survival (OS) were compared according to the pathologic node positivity (ypN- vs. ypN+). Among a total of 165 patients with MIBC who received neoadjuvant chemotherapy involving GP, 118 underwent radical cystectomy. In 46 patients with $\mathrm{ypN}+$ disease, DFS and OS were evaluated according to administration of adjuvant GP.

Results: After neoadjuvant chemotherapy and radical cystectomy, $41 \%$ of patients had ypN+ disease, which showed significantly shorter DFS (median, 7.4 months; 95\% confidence interval [CI], 5.3-9.6 months) and OS (median, 20.0 months; $95 \% \mathrm{Cl}, 13.4-26.6$ months) compared to those with ypN- disease. The patients with ypN+ disease had a high risk of recurrence or death, regardless of the administration of adjuvant chemotherapy or adjuvant regimen. Conclusions: Within the limitations of this retrospective study, MIBC patients with ypN+ disease despite neoadjuvant chemotherapy and radical cystectomy had a poor prognosis. Further studies involving novel, effective adjuvant treatment including immunotherapy agents are needed to reduce the high risk of recurrence or death in these patients. (Korean J Urol Oncol 2020;18:194-200)
\end{abstract}

Key Words: Urinary bladder neoplasms $\cdot$ Neoadjuvant chemotherapy $\cdot$ Lymph nodes $\cdot$ Cystectomy

Received June 12, 2020, Revised July 28, 2020,

Accepted August 10, 2020

Corresponding Author: Se Hoon Park

Division of Hematology-Oncology, Department of Medicine, Samsung Medical Center, Sungkyunkwan University School of Medicine, 81 Irwon-ro, Gangnam-gu, Seoul 06351, Korea

E-mail: hematoma@skku.edu

Tel: +82-2-3410-3459, Fax: +82-2-3410-1754

ORCID: https://orcid.org/0000-0001-5084-9326

\section{INTRODUCTION}

Urothelial carcinoma of the bladder, a cancer involving the transitional epithelium of the urinary bladder, is the seventh most common malignancy in Korea. ${ }^{1}$ Bladder cancer (BC) presents as superficial (i.e., nonmuscle invasive), mus-

This is an Open Access article distributed under the terms of the Creative Commons Attribution Non-Commercial License (http://creativecommons.org/licenses/by-nc/4.0/) which permits unrestricted non-commercial use, distribution, and reproduction in any medium, provided the original work is properly cited. 2020 (C) Copyright The Korean Urological Oncology Society and The Korean Prostate Society. All Rights Reserved. 
cle invasive BC (MIBC), or metastatic disease. For patients with MIBC or locally advanced disease, radical cystectomy with bilateral pelvic lymph node dissection (PLND) forms the backbone of management. Nevertheless, for those with high-risk features such as pT3-T4 or $\mathrm{pN}+$ disease, the probability of survival after cystectomy remains very poor. To improve the outcome, neoadjuvant chemotherapy has been proposed for patients with clinical stage T2-T4aN0M0 MIBC who are candidates for radical surgery. Two large randomized trials using cisplatin-based combination chemotherapy. ${ }^{2,3}$ and a meta-analysis ${ }^{4}$ suggest that neoadjuvant chemotherapy for patients with MIBC provides a significant survival benefit than surgery alone.

Since the publication of evidence-based guidelines, ${ }^{5-7}$ neoadjuvant chemotherapy has come to be standard clinical practice in patients with MIBC. The rationale for perioperative chemotherapy is on the basis of response in patients with locally advanced or metastatic BC. Cisplatin-based chemotherapy showed efficacy, with a median survival of approximately 15 months, with responses in $40 \%-60 \%$ of patients. ${ }^{8}$ Downstaging of the tumor may show the efficacy of neoadjuvant chemotherapy, especially in patients who have a pathologic complete response (pCR) or who are $\leq$ pT1N0M0 after treatment. As expected, on the other hand, some patients present with positive lymph node (LN) even after neoadjuvant chemotherapy and complete resection. Though insufficient initial response could indicate chemotherapy refractoriness, a retrospective study found improved survival outcomes with adjuvant chemotherapy in $\mathrm{ypN}+$ MIBC. ${ }^{9}$

Since a multidisciplinary urological cancer team was put in place at our institution in 2010, use of neoadjuvant chemotherapy followed by radical cystectomy in medically fit patients with MIBC has been the standard of care. All eligible patients (i.e., cT2-T4aN0M0, glomerular filtration rate [GFR] $>60 \mathrm{~mL} / \mathrm{min}$ ) receive 2 or more cycles of gemcitabine and cisplatin (GP) chemotherapy before surgery. The objective of the present study is to retrospectively evaluate clinical outcomes in patients with $\mathrm{ypN}+\mathrm{MIBC}$ after neoadjuvant chemotherapy and radical surgery.

\section{MATERIALS AND METHODS}

During the study period from 2010 to 2014,337 patients underwent radical cystectomy for $\mathrm{BC}$ in the participating center, and 301 of them were found to have MIBC at the time of surgery. Upon review of medical records, 165 patients were identified who were treated with neoadjuvant chemotherapy involving GP (Fig. 1). Among 118 patients treated with neoadjuvant GP followed by radical cystectomy, 5 additional patients were excluded: M1 $(n=2)$, no PLND ( $n=2)$, and lost to follow-up $(n=1)$. Finally, 67 and 46 patients who had ypN- and ypN+ disease, respectively, after radical cystectomy were evaluated in the present study. Our genitourinary pathologist (GYK) reviewed all pathology specimens to ensure the samples included adequate numbers of LNs. Data were collected in a standardized manner by means of a previously approved form and by a single trained investigator (JHC). All patients provided written informed consent for neoadjuvant chemotherapy as well as surgery. This retrospective study was performed in accordance with the Declaration of Helsinki and approved by the Institutional Review Board (IRB) of Samsung Medical Center (IRB No. 2017-10-008).

Patients were required to have MIBC without LN or distant metastasis (i.e., cT2-T4aN0M0), performance status of 0 or 1 , and adequate major organ functions including GFR

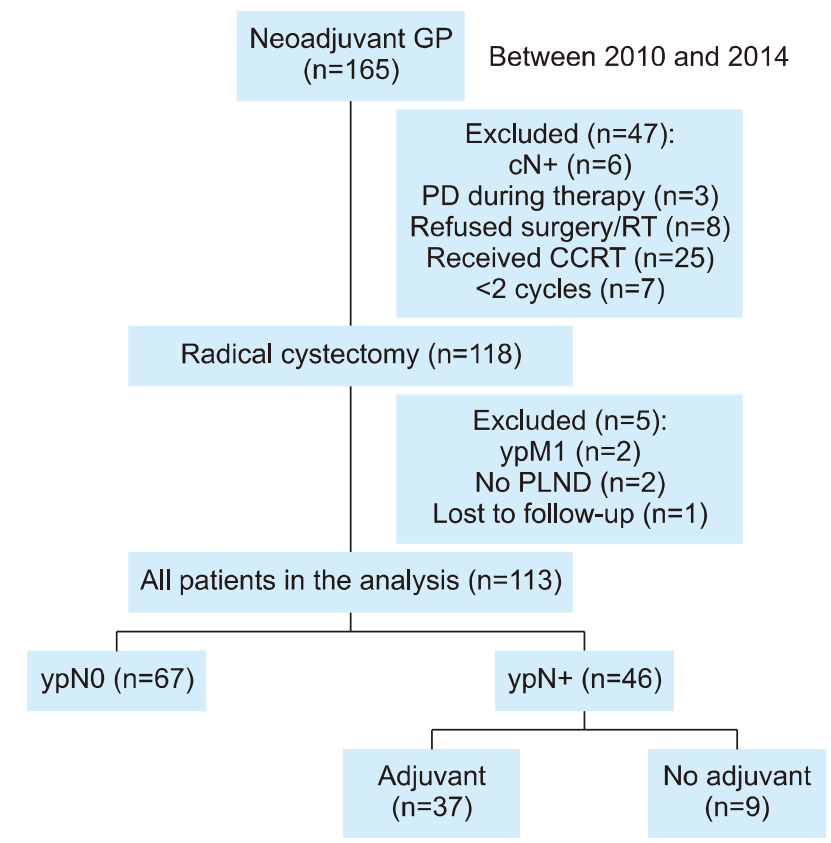

Fig. 1. CONSORT (consolidated standards for reporting of trials) diagram for study population. GP: gemcitabine and cisplatin, CCRT: concurrent chemoradiotherapy, PLND: pelvic lymph node dissection. 
$>60 \mathrm{~mL} / \mathrm{min}$. Neoadjuvant GP consisted of 2 or more cycles, depending on the schedule of surgery, of gemcitabine $1,000 \mathrm{mg} / \mathrm{m}^{2}$ on days 1,8 , and 15 plus cisplatin $60 \mathrm{mg} / \mathrm{m}^{2}$ on day 1. Chemotherapy was repeated every 4 weeks on an outpatient basis. Supportive care including administration of pre- and postcisplatin hydration and use of hematopoietic growth factors, antiemetics, and analgesics was given if judged appropriate by the treating oncologists. When a patient completed the planned neoadjuvant GP cycles, in the absence of disease progression during or after chemotherapy, radical cystectomy with bilateral PLND was offered according to the institutional standards. If the patient refused surgery, an alternative treatment involving bladder-preserving concurrent chemoradiotherapy was performed. After surgery, adjuvant chemotherapy was not routinely administered but was given to some patients at the discretion of the treating oncologists. All patients were followed approximately every 3 months for recurrence and survival.

The primary endpoint was duration of disease-free survival (DFS), which was calculated from the date of surgery to the date of disease recurrence (including distant metastases, local and regional recurrence) or death, whichever occurred first. The secondary endpoint of the study was overall survival (OS). All statistical analyses were performed

Table 1. Baseline characteristics of all patients

\begin{tabular}{|c|c|c|c|}
\hline Characteristic & All patients $(\mathrm{n}=113)$ & ypN0 $(n=67)$ & $\mathrm{ypN}+(\mathrm{n}=46)$ \\
\hline Age (yr), median (range) & $68(40-83)$ & $66(40-83)$ & $68(51-81)$ \\
\hline \multicolumn{4}{|l|}{ Sex } \\
\hline Male & $94(83)$ & $56(84)$ & $38(83)$ \\
\hline Female & $19(17)$ & $11(16)$ & $8(17)$ \\
\hline \multicolumn{4}{|l|}{ Stage at diagnosis } \\
\hline Superficial & $28(25)$ & $18(27)$ & $10(22)$ \\
\hline Muscle invasive & $85(75)$ & $49(73)$ & $36(78)$ \\
\hline \multicolumn{4}{|l|}{$\mathrm{T}$ stage at surgery } \\
\hline 2 & $68(60)$ & $46(69)$ & $22(48)$ \\
\hline 3 & $37(33)$ & $18(27)$ & $19(41)$ \\
\hline $4 \mathrm{a}$ & $8(7)$ & $3(4)$ & $5(11)$ \\
\hline \multicolumn{4}{|l|}{ Neoadjuvant cycles } \\
\hline 2 & $65(58)$ & $41(61)$ & $24(52)$ \\
\hline 3 & $41(36)$ & $22(33)$ & $19(41)$ \\
\hline 4 & $7(6)$ & $4(6)$ & $3(7)$ \\
\hline Total neoadjuvant cycles & 281 & 164 & 117 \\
\hline \multicolumn{4}{|l|}{ Tumor grade } \\
\hline 2 & $26(23)$ & $20(30)$ & $6(13)$ \\
\hline 3 & $87(77)$ & $47(70)$ & $40(87)$ \\
\hline \multicolumn{4}{|l|}{ Pathologic $\mathrm{T}$ stage } \\
\hline 0 (including $\mathrm{Ta} / \mathrm{Tis}$ ) & $23(20)$ & $22(33)$ & $1(2)$ \\
\hline 1 & $19(17)$ & $15(22)$ & $4(9)$ \\
\hline 2 & $25(22)$ & $15(22)$ & $10(22)$ \\
\hline 3 or 4 & $46(41)$ & $15(22)$ & $31(67)$ \\
\hline \multicolumn{4}{|l|}{ Pathologic $\mathrm{N}$ stage } \\
\hline 0 & $67(59)$ & $67(100)$ & \\
\hline 1 & $23(20)$ & - & $23(50)$ \\
\hline 2 & $21(19)$ & - & $21(46)$ \\
\hline 3 & $2(2)$ & - & $2(4)$ \\
\hline \multicolumn{4}{|l|}{$\mathrm{N}$ ratio, median (range) } \\
\hline Removed & $20(3-47)$ & $20(3-47)$ & $31(4-46)$ \\
\hline Positive & $0(0-25)$ & $0(0)$ & $1.5(1-25)$ \\
\hline Ratio & $0(0-0.95)$ & $0(0)$ & $0.1 \quad(0.02-0.95)$ \\
\hline Lymphovascular invasion & $35(31)$ & $8(12)$ & $27(59)$ \\
\hline
\end{tabular}

Values are presented as median (range) or number (\%). 
A

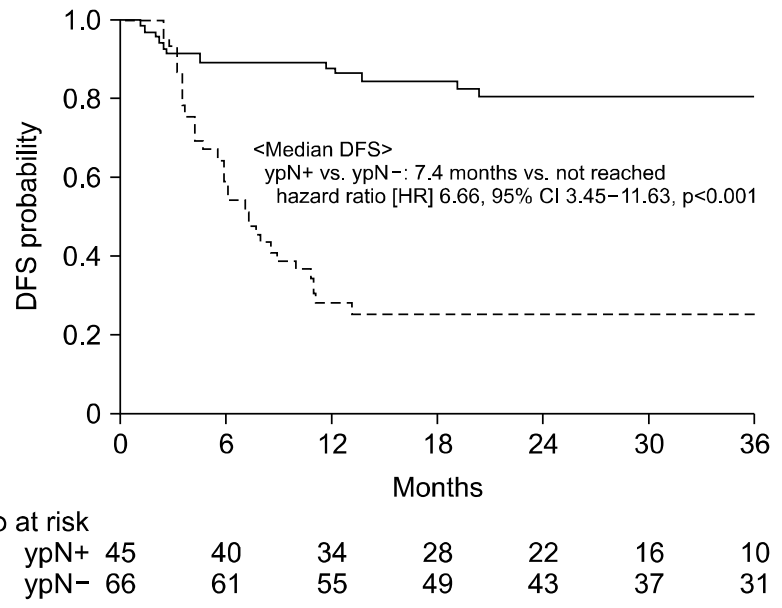

B

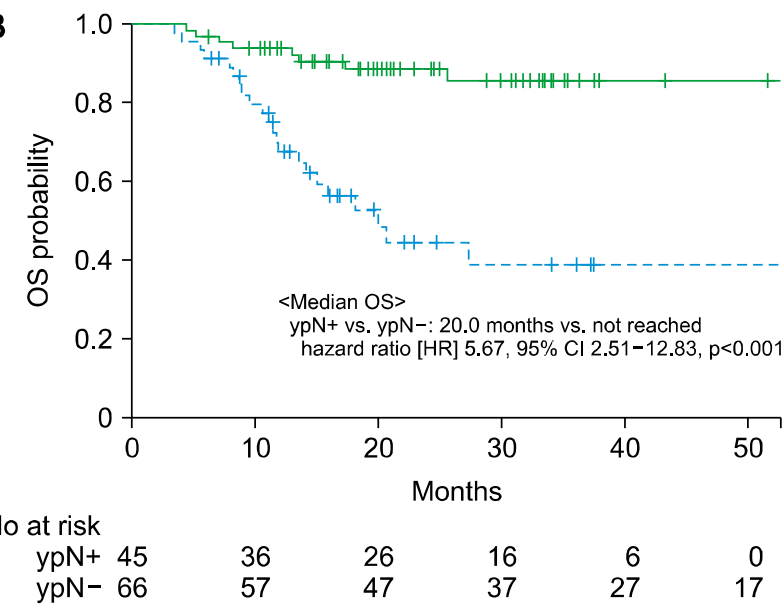

Fig. 2. (A) Disease-free survival (DFS) between patients with ypN- (solid line) and ypN+ (dotted line) diseases. (B) Overall survival (OS) between patients with ypN- (green line) and ypN+ (blue line) diseases.

using R for Windows v3.3.1 software (R Core Team, Vienna, Austria; http://www.Rproject.org). The Kaplan-Meier method was used to estimate DFS and OS.

\section{RESULTS}

As shown in Fig. 1, 113 MIBC patients were included for analysis. Baseline characteristics of all patients are listed in Table 1. Patients were predominantly male (83\%), and the median age at the time of surgery was 68 years (range, 40-83 years). One-third of the patients underwent ileal neobladder after radical cystectomy, and the median number of LNs removed during PLND was 20 (range, 3-47). A median of 2 cycles (range, 2-5) of neoadjuvant chemotherapy was administered. Overall, neoadjuvant GP was generally well-tolerated. The patients with $\mathrm{ypN}+$ disease had significantly higher tumor grade $3(87.0 \%$ vs. $70.1 \%, \mathrm{p}=0.04)$ and lymphovascular invasion rate $(58.7 \%$ vs. $11.9 \%, \mathrm{p}=0.001)$ than patients with ypN0 disease.

According to the surgical pathology reports, complete response (CR, ypT0N0) and down-staging (<ypT1N0) were achieved in 22 patients $(20 \%)$ and 37 patients (33\%), respectively. Pathologic response was not significantly influenced by age, sex, clinical $\mathrm{T}$ stage, or duration of neoadjuvant chemotherapy. Among 46 patients with ypN+ disease, adjuvant chemotherapy was given to 37 patients after surgery: GP $(n=28)$ and paclitaxel $(n=9)$. At the time of data collection, with a median follow-up duration of 30 months, 46 patients $(41 \%)$ experienced recurrence and 30 patients
(27\%) had died.

Of the 113 patients analyzed in the study, the median DFS and OS were not reached. Patients with ypN+ disease had significantly shorter DFS (median, 7.4 months; 95\% confidence interval [CI], 5.3-9.6 months) compared to those with ypN- disease (hazard ratio [HR], 6.66; 95\% CI 3.4511.63; $\mathrm{p}<0.001)$. The Kaplan-Meier estimates for DFS in patients with $\mathrm{ypN}-$ and $\mathrm{ypN}+$ disease are illustrated in Fig. 2A. OS also was shorter in patients with ypN+ disease (median, 20.0 months; 95\% CI, 13.4-26.6 months) than ypN- patients (HR, 5.67; 95\% CI, 2.51-12.83; p<0.001) (Fig. 2B). Notably, no differences were observed in DFS whether the ypN+ patients received adjuvant chemotherapy or not (median, 7.8 vs. 5.6 months; $\mathrm{p}=0.843$ ). In 37 patients who were treated with adjuvant chemotherapy, although not statistically significant, median DFS was longer for GP (8.7 months) than paclitaxel (5.9 months, $\mathrm{p}=0.663$ ).

\section{DISCUSSION}

Thirty percent of $\mathrm{BC}$ patients are diagnosed with MIBC, and about $50 \%$ of MIBC patients will develop distant metastases within the first 2 years after treatment. ${ }^{10}$ The perioperative morbidity rate was low when radical cystectomy was performed at a center of excellence. ${ }^{10}$ Despite an aggressive surgical approach, however, up to $50 \%$ of patients developed recurrence, suggesting that a significant proportion of the patients had micrometastases at the time of surgery. The rationale for utilization of perioperative che- 
motherapy is on the basis of the response in patients with locally advanced or metastatic BC: cisplatin-based chemotherapy has demonstrated efficacy responses in $40 \%-60 \%$ of such patients. ${ }^{8}$ A meta-analysis of data from 11 randomized trials has demonstrated that MIBC is most sensitive to cisplatin-based neoadjuvant chemotherapy, with a 5\% net benefit in 5-year OS. ${ }^{4}$ Commonly used cisplatin-based neoadjuvant chemotherapy regimens include M-VAC (methotrexate, vinblastine, doxorubicin, and cisplatin), CMV (methotrexate, vinblastine, and cisplatin), and GP (gemcitabine and cisplatin). Clinical practice guidelines recommend cisplatinbased neoadjuvant chemotherapy prior to cystectomy for patients with MIBC. ${ }^{5-7}$ Patients with MIBC who achieve a ypCR (pT0N0 stage) or whose condition is down-staged to nonmuscle invasive disease after neoadjuvant chemotherapy demonstrate longer OS than those who fail to achieve pCR or are not down-staged. ${ }^{11}$ For patients with residual muscle invasive disease (ypT2 or higher) or LN-positive disease $(\mathrm{ypN}+)$ at cystectomy, the median survival is only 3.4 and 2.4 years, respectively. Likewise, DFS at 2 or 3 years correlates with the 5-year OS of patients undergoing radical cystectomy for MIBC. ${ }^{12}$ In a study of 52 patients with $\mathrm{BC}$ and pathologically confirmed LN-positive disease, response to neoadjuvant platinum-based chemotherapy resulted in improved survival, and LN status was more important than local tumor status. ${ }^{13}$

In clinical practice outside the clinical trial setting, it has been reported that $<20 \%$ of patients have received neoadjuvant chemotherapy prior to radical cystectomy. ${ }^{14}$ Many patients with MIBC choose to undergo definitive surgery (i.e., radical cystectomy) first, and defer chemotherapy; patients at high risk for recurrence on the basis of pathologic staging could consider adjuvant chemotherapy given its potential to prevent recurrence. However, unlike the benefits that shown with neoadjuvant chemotherapy, meta-analyses found no evidence that adjuvant chemotherapy improved survival outcomes, even in the highest-risk patients with pathologic extravesical and/or node-positive disease. ${ }^{4,15}$ As one of the largest randomized trials ever reported for adjuvant chemotherapy in patients with $\mathrm{MIBC},{ }^{16}$ the EORTC (European Organisation for Research and Treatment of Cancer) trial was closed after recruitment of 284 of the planned 660 patients. In that study, 284 patients with pT3pT4 or N+ M0 MIBC were randomized to receive either immediate versus deferred cisplatin-based combination chemotherapy after cystectomy. Immediate chemotherapy significantly extended progression-free survival compared with deferred chemotherapy (HR, 0.54; 95\% CI, 0.4-0.73) $(\mathrm{p}<0.0001)$. However, there is no significant OS benefit in patients with immediate chemotherapy compared with deferred chemotherapy (HR, 0.78; 95\% CI, 0.56-1.08) ( $\mathrm{p}=0.13)$. Thus, the benefit and usefulness of cisplatin-based adjuvant chemotherapy remain controversial.

As mentioned above, cases with residual disease despite neoadjuvant chemotherapy are more challenging. In the prospective SWOG trial, ${ }^{3}$ most patients with $\mathrm{ypN}+$ disease according to surgical pathology results had a grim prognosis. Based on the high recurrence rate in $\mathrm{ypN}+$ disease, patients often are offered additional adjuvant chemotherapy, either with cisplatin-based or potentially non-cross-resistant agents, in an attempt to improve the otherwise poor outcomes. One may argue that further use of cisplatin-based chemotherapy in the adjuvant treatment after neoadjuvant chemotherapy is limited because of the resistance problem as well as patient-related factors including performance status and renal function. In the present study, 41\% (46 of 113) of patients were found to have $\mathrm{ypN}+$ disease according to surgical pathology results. The rate of node positivity is quite high in the context of prior reports generally suggesting a LN positivity rate of about $20 \%-25 \%{ }^{17,18}$ We observed that the median DFS was significantly shorter in those with persistent nodal disease despite neoadjuvant chemotherapy and radical cystectomy (HR, 6.66; 95\% CI, 3.45-11.63) $(\mathrm{p}<0.001)$. Not surprisingly, because of the lack of evidence to guide the treatment of patients with ypN+ disease, $80 \%$ (37 of 46) of patients received adjuvant chemotherapy but still had a high recurrence risk and death, regardless of adjuvant regimen.

This study has several limitations. First, interpretation of the study should be cautious given small sample size of the study and the retrospective nature. Second, we did not assess neoadjuvant GP dose density, dose adjustment, or chemotherapy-related toxicity. Third, the study is inconsistent with the National Comprehensice Cancer Institute and American Urological Association guideline recommendations for preferred 4 cycles of GP and at minimum 3 cycles prior to cystectomy. The reason for this result is that the first is the limit of the retrospective study, and the second is the 
4 cycles of GP recommended for our urology cancer institution team; however, it is presumed to be due to the surgeon's operation schedule and operating room availability. Fourth, there is a wide variation in clinical practice among centers and treating physicians. In a retrospective study involving 150 patients with ypN+ disease, ${ }^{9}$ there was a significant DFS benefit $(\mathrm{p}=0.02)$ in 37 patients who were treated with adjuvant chemotherapy. Even in a recent retrospective study of 81 patients with MIBC and pathologically confirmed LN metastases, patients treated with neoadjuvant chemotherapy had significantly worse 3-year recurrencefree survival $(26 \%)$ compared to chemotherapy-naïve LNpositive patients subsequently treated with adjuvant chemotherapy $(60 \%) .{ }^{19} \mathrm{~A}$ more recent study also suggested that adjuvant chemotherapy after neoadjuvant chemotherapy and radical cystectomy may extend OS among patients with MIBC. ${ }^{20}$ Lastly, in some cases, PLND can be curative, especially in patients with a low $\mathrm{LN}$ ratio. ${ }^{21}$ In addition to node positivity, the total number of LNs removed, or LN ratio, is an indirect measure of the extent of PLND, and it may serve as an important factor in MIBC surgery. ${ }^{22}$ Even in patients with $\mathrm{ypN}+$ disease, removing more LNs could result in clearance of micrometastatic cancer foci that are not detected by pathology assessment. A portion of the longterm DFS observed in some patients with $\mathrm{ypN}+$ disease can likely be attributed to the clearance of such micrometastases.

The promising results of checkpoint inhibitors in the advanced setting increased the application of immunotherapy also in the neoadjuvant setting. Several clinical trials are ongoing, and 2 ones have already been reported. ABACUS study is a single arm, phase 2 neoadjuvant trial with atezolizumab, administered for 2 cycles before surgery. This study reported a promising CR rate of $29 \%$ (18/62 patients). ${ }^{23}$ In the PURE (Prospective Urban Rural Epidemiology) trial, 3 cycles of pembrolizumab were administered after transurethral resection and before radical cystectomy to 50 patients. This trial was described in $42 \%$ of patients. ${ }^{24}$ Many trials explore the combinations checkpoint inhibitors with standard chemotherapy. The preliminary results of a PAIR (phase $1 \mathrm{~b} / 2$ neoadjuvant) trial, a combination with pembrolizumab plus chemotherapy was promisingly reported in $60 \%$ of patients at ESMO (European Society for Medical Oncology) $2018 .^{25}$ Future trials will have to define which is the optimal drug choice and the role of immunotherapy.

\section{CONCLUSIONS}

In summary, this study demonstrated that $\mathrm{ypN}+\mathrm{MIBC}$ patients have a grim prognosis after neoadjuvant chemotherapy and radical cystectomy. Further prospective studies are needed to improve outcomes, including more effective adjuvant regimens. The ongoing rapid knowledge expansion about the biology of $\mathrm{BC}$ and new data regarding the efficacy of immunotherapy agents in this disease will promise noteworthy changes in the future direction of adjuvant therapies.

\section{CONFLICTS OF INTEREST}

The authors claim no conflicts of interest.

\section{REFERENCES}

1. Roscigno M, Shariat SF, Freschi M, Margulis V, Karakiewizc P, Suardi N, et al. Assessment of the minimum number of lymph nodes needed to detect lymph node invasion at radical nephroureterectomy in patients with upper tract urothelial cancer. Urology 2009;74:1070-4.

2. International Collaboration of Trialists; Medical Research Council Advanced Bladder Cancer Working Party (now the National Cancer Research Institute Bladder Cancer Clinical Studies Group); European Organisation for Research and Treatment of Cancer Genito-Urinary Tract Cancer Group; Australian Bladder Cancer Study Group; National Cancer Institute of Canada Clinical Trials Group; Finnbladder, et al. International phase III trial assessing neoadjuvant cisplatin, methotrexate, and vinblastine chemotherapy for muscle invasive bladder cancer: long-term results of the BA06 30894 trial. J Clin Oncol 2011;29: 2171-7.

3. Grossman HB, Natale RB, Tangen CM, Speights VO, Vogelzang NJ, Trump DL, et al. Neoadjuvant chemotherapy plus cystectomy compared with cystectomy alone for locally advanced bladder cancer. N Engl J Med 2003; 349:859-66.

4. Advanced Bladder Cancer Meta-analysis Collaboration. Neoadjuvant chemotherapy in invasive bladder cancer: update of a systematic review and meta-analysis of individual patient data advanced bladder cancer (ABC) metaanalysis collaboration. Eur Urol 2005;48:202-5; discussion 205-6. 
5. Montie JE, Clark PE, Eisenberger MA, El-Galley R, Greenberg RE, Herr HW, et al. Bladder cancer. J Natl Compr Canc Netw 2009;7:8-39.

6. Witjes JA, Comperat E, Cowan NC, De Santis M, Gakis $\mathrm{G}$, Lebret $\mathrm{T}$, et al. EAU guidelines on muscle-invasive and metastatic bladder cancer: summary of the 2013 guidelines. Eur Urol 2014;65:778-92.

7. Bellmunt J, Albiol S, Kataja V, Group EGW. Invasive bladder cancer: ESMO clinical recommendations for diagnosis, treatment and follow-up. Ann Oncol 2009;20 Suppl 4:79-80.

8. Kassouf W, Agarwal PK, Grossman HB, Leibovici D, Munsell MF, Siefker-Radtke A, et al. Outcome of patients with bladder cancer with $\mathrm{pN}+$ disease after preoperative chemotherapy and radical cystectomy. Urology 2009;73: 147-52.

9. van Rhijn BW, Vis AN, van der Kwast TH, Kirkels WJ, Radvanyi F, Ooms EC, et al. Molecular grading of urothelial cell carcinoma with fibroblast growth factor receptor 3 and MIB-1 is superior to pathologic grade for the prediction of clinical outcome. J Clin Oncol 2003;21: 1912-21.

10. Witjes JA, Lebret T, Comperat EM, Cowan NC, De Santis M, Bruins HM, et al. Updated 2016 EAU guidelines on muscle-invasive and metastatic bladder cancer. Eru Urol 2017;71:462-75.

11. Petrelli F, Coinu A, Cabiddu M, Ghilardi M, Vavassori I, Barni S. Correlation of pathologic complete response with survival after neoadjuvant chemotherapy in bladder cancer treated with cystectomy: a meta-analysis. Eur Urol 2014; 65:350-57.

12. Sonpavde G, Khan MM, Lerner SP, Svatek RS, Novara G, Karakiewicz PI, et al. Disease-free survival at 2 or 3 years correlates with 5-year overall survival of patients undergoing radical cystectomy for muscle invasive bladder cancer. J Urol 2011;185:456-61.

13. Nieuwenhuijzen JA, Bex A, Meinhardt W, Kerst JM, Schornagel JH, Van Tinteren H, et al. Neoadjuvant methotrexate, vinblastine, doxorubicin and cisplatin for histologically proven lymph node positive bladder cancer. J Urol 2005; 174:80-5.

14. Raj GV, Karavadia S, Schlomer B, Arriaga Y, Lotan Y, Sagalowsky A, et al. Contemporary use of perioperative cisplatin-based chemotherapy in patients with muscle-invasive bladder cancer. Cancer 2011;117:276-82.

15. Meeks JJ, Bellmunt J, Bochner BH, Clarke NW, Daneshmand S, Galsky MD, et al. A systematic review of neoadjuvant and adjuvant chemotherapy for muscle-in- vasive bladder cancer. Eur Urol 2012;62:523-33.

16. Sternberg CN, Skoneczna I, Kerst JM, Albers P, Fossa SD, Agerbaek $\mathrm{M}$, et al. Immediate versus deferred chemotherapy after radical cystectomy in patients with pT3-pT4 or N+ M0 urothelial carcinoma of the bladder (EORTC 30994): an intergroup, open-label, randomised phase 3 trial. Lancet Oncol 2015;16:76-86.

17. Hautmann RE, de Petriconi RC, Pfeiffer C, Volkmer BG. Radical cystectomy for urothelial carcinoma of the bladder without neoadjuvant or adjuvant therapy: long-term results in 1100 patients. Eur Urol 2012;61:1039-47.

18. Stein JP, Lieskovsky G, Cote R, Groshen S, Feng AC, Boyd S, et al. Radical cystectomy in the treatment of invasive bladder cancer: long-term results in 1,054 patients. J Clin Oncol 2001;19:666-75.

19. Cha EK, Sfakianos JP, Sukhu R, Yee AM, Sjoberg DD, Bochner BH. Poor prognosis of bladder cancer patients with occult lymph node metastases treated with neoadjuvant chemotherapy. BJU Int 2018;122:627-32.

20. Seisen T, Jamzadeh A, Leow JJ, Roupret M, Cole AP, Lipsitz SR, et al. Adjuvant chemotherapy vs observation for patients with adverse pathologic features at radical cystectomy previously treated with neoadjuvant chemotherapy. JAMA Oncol 2018;4:225-9.

21. Cancer Genome Atlas Research Network. Comprehensive molecular characterization of urothelial bladder carcinoma. Nature 2014;507:315-22.

22. Raggi D, Miceli R, Sonpavde G, Giannatempo P, Mariani $\mathrm{L}$, Galsky MD, et al. Second-line single-agent versus doublet chemotherapy as salvage therapy for metastatic urothelial cancer: a systematic review and meta-analysis. Ann Oncol 2016;27:49-61.

23. Powles T, Rodriguez-Vida A, Duran I, Crabb SJ, Van Der Heijden MS, Font Pous A, et al. A phase II study investigating the safety and efficacy of neoadjuvant atezolizumab in muscle invasive bladder cancer (ABACUS). J Clin Oncol 2018;36(15_suppl):4506.

24. Gallina A, Necchi A, Anichini A, Raggi D, Briganti A, Massa S, et al. Pembrolizumab as neoadjuvant therapy before radical cystectomy in patients with muscle-invasive urothelial bladder carcinoma (PURE-01): an open-label, single-artm, phase II study. J Clin Oncol 2018;36:3353-60.

25. Hoimes CJ, Albany C, Hoffman-Censits J, Fleming MT, Trabulsi E, Picus J, et al. A phase $1 \mathrm{~b} / 2$ study of neoadjuvant pembrolizumab (pembro) and chemotherapy for lically advanced urothelial cancer (UC) [abstract]. Ann Oncol 2018;29(suppl_8):VII726. 